\title{
In vitro Glucuronidation of Estradiol-17 $\beta$ by Microsomes Prepared using Liver Biopsy Specimens from Dairy Cows
}

\author{
Hiroya KADOKAWA ${ }^{1)}$, Junya MATSUMOTO ${ }^{2)}$ and Hiroshi YOKOTA ${ }^{2)}$ \\ ${ }^{1)}$ Department of Veterinary Medicine, Faculty of Agriculture, Yamaguchi University, 1677-1 Yoshida, Yamaguchi 753-8515 and \\ ${ }^{2}$ School of Veterinary Medicine, Rakuno Gakuen University, Ebetsu, Hokkaido 069-8501, Japan
}

(Received 31 August 2006/Accepted 23 January 2007) ABSTRACT. Increased hepatic metabolism of estradiol may cause weakened estrous behavior in lactating dairy cows, but this hypothesis
must be examined further, especially through diachronic study of the hepatic estradiol-17 $\beta$ glucuronidation activity of uridine diphosphate
(UDP)-glucuronosyltransferases. Therefore, in order to develop a new tool for this purpose, we attempted to conduct biopsy of the livers
of dairy cows with the aid of ultrasonography and to measure the UDP-glucuronosyltransferase activities of microsomes of the specimens
using in vitro glucuronidation followed by HPLC analysis. We were able to measure the activities of the microsomes prepared from the
liver biopsy, and the results seemed reliable. Therefore, this method may become a new tool in clinical studies to detect estradiol-17 $\beta$
glucuronidation activity.

KEY WORDS: Estrogen metabolism, Estrus, UDP-glucuronosyltransferase.

J. Vet. Med. Sci. 69(5): 557-559, 2007

Wiltbank et al. [12] recently proposed the hypothesis that increased liver blood flow resulting from elevated feed intake increases estrogen metabolism and may cause low circulating concentrations of estradiol-17 $\beta$. This may cause changes in reproduction, such as decreased conception rate, increased pregnancy loss, increased multiple ovulation rate, and decreased behavioral estrus. This hypothesis seems very significant. Therefore, further study of hepatic steroid metabolism in dairy cows is required in order to gain a better understanding and to develop a new non-hormonal strategy to improve reproduction in dairy cows $[4,7]$ because there are a limited number of hormonal treatments available at the moment [12].

The uridine diphosphate (UDP)-glucuronosyltransferase family is the most important group of hepatic enzymes for the main pathway of estrogen metabolism. This enzyme family catalyzes biotransforming lipid-soluble xenobiotics and endobiotics (e.g., estradiol-17 $\beta$ ) to render them more water-soluble by glucuronide conjugation, and the resultant glucuronidated xenobiotics and endobiotics can be easily excreted into bile and urine [5]. UDP-glucuronosyltransferase activity has been studied mainly in biochemical studies using samples collected from slaughtered laboratory animals [8]. However, the system for measuring UDP-glucuronosyltransferase activity may also be used for clinical studies of dairy cows. Thus, we conducted a preliminary trial of biopsy of liver specimens from dairy cows with the aid of ultrasonography and measurement of UDP-glucuronosyltransferase activity in microsomes from the biopsy specimen using in vitro glucuronidation followed by highperformance liquid chromatographic (HPLC) analysis.

Two non-lactating, non-pregnant multiparous Holstein dairy cows (parity $=3$ or 4 ) were housed in a tie-stall barn of the National Agricultural Research Center for Hokkaido Region. They were provided with free access to their diet (grass hay; $83.3 \%$ dry matter, $1.92 \mathrm{Mcal} / \mathrm{kg}$ of metaboliz- able energy, and $6.7 \%$ crude protein) to meet dry cow requirements according to the Japanese Feeding Standard [1]. One of the cows was euthanized by exsanguination immediately after sedation with xylazine $(0.3 \mathrm{mg} / \mathrm{kg}$ body weight, intravenously) and before biopsy (the procedure is described in detail later), and its liver was sampled under visual observation within 20 min of death. The microsome prepared from the liver sample of the euthanized cow was aliquoted and frozen at $-80^{\circ} \mathrm{C}$ until utilization for the following purposes: (1) calculating the parameters of the kinetics of UDP-glucuronosyltransferase for estradol-17 $\beta$, maximum velocity, and Michaelis constant values; (2) 4 replicates of triplicate analysis in order to determine the inter- and intra-assay coefficients of variation (CVs) among assays performed on different days; and (3) comparison of the UDP-glucuronosyltransferase activity with the microsome prepared from the biopsy specimen. Biopsy was performed 5 times on the remaining cow to determine the intra-biopsy CV. All samples were immediately frozen in liquid nitrogen, brought to the laboratory, and stored at $-80^{\circ} \mathrm{C}$ until analysis.

The biopsy method utilized for these cows was previously reported by Mohamed et al. [9]. Briefly, the right abdominal wall from the 7 th to the 12 th intercostal spaces was prepared surgically, the cows was slightly sedated with xylazine $(0.07 \mathrm{mg} / \mathrm{kg}$ body weight, intravenously) in order to obtain an adequate amount of restraint, and the abovementioned region was infiltrated with $10 \mathrm{ml}$ of $2 \%$ procaine hydrochloride. Ultrasonic coupling gel was liberally applied to the wet skin. A $3.5-\mathrm{MHz}$ linear transducer connected to an ultrasound scanner (Model RT 2600, Yokogawa Medical Systems, Tokyo, Japan) was held firmly against the body wall to localize the liver (Fig. 1). After an entry site was chosen, the skin was incised with the tip of a No. 20 scalpel blade. Then, a 14-gauge $\times 150 \mathrm{~mm}$ biopsy needle for the diagnosis of fatty liver (305056, Kurita- 


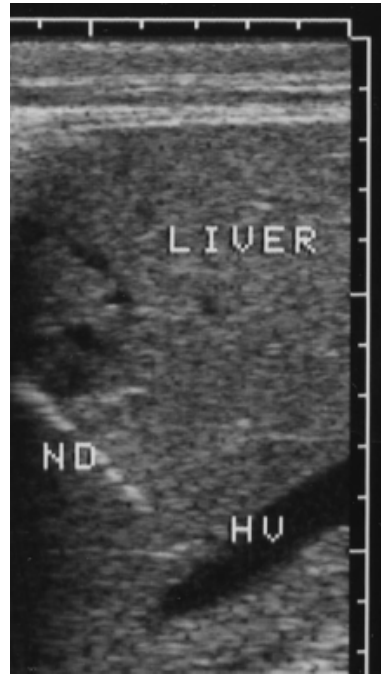

Fig. 1. Sonograph of liver biopsied for one of the dairy cows. ND: the tip of the biopsy needle. HV: the hepatic portal vein.
(A)

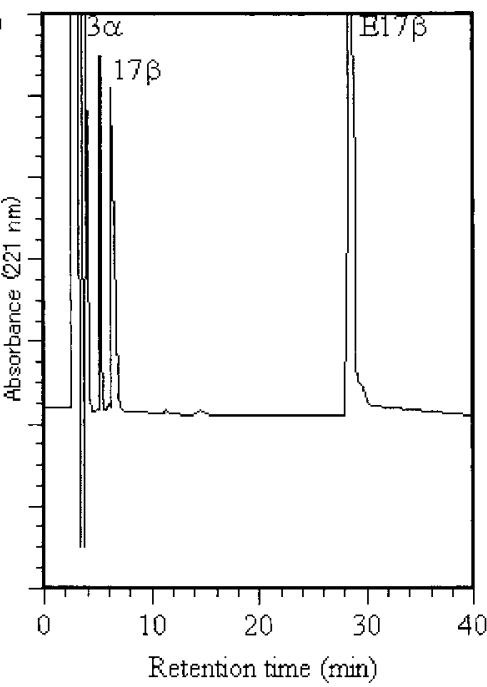

(B)

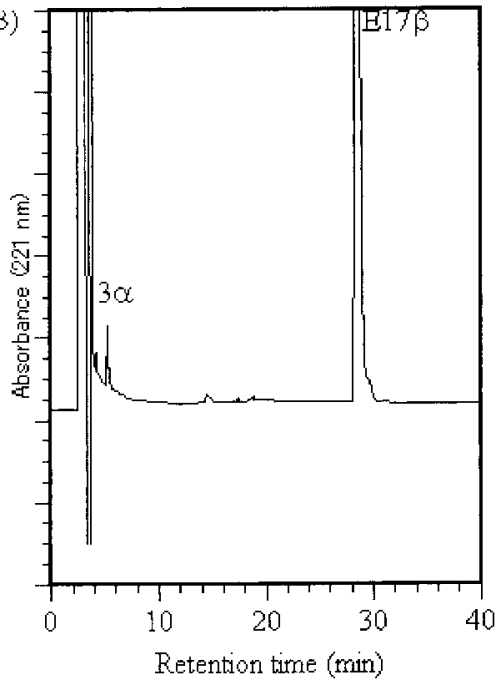

Fig. 2. Typical HPLC chromatograms of (a) the standard mix solution and (b) the resultant enzyme reaction products of estradiol glucuronidation catalyzed by UDP-glucuronosyltransferases in the microsomes prepared from the biopsied liver of a dairy cow. $3 \alpha$ : estradiol-3 $\alpha$-glucuronide (main products). 17 $\beta$ : estradiol-17 $\beta$-glucuronide. E17 $\beta$ : estradiol-17 $\beta$ as the substrate for reaction.

Chushasin Seisakusho, Tokyo) was advanced through the skin into the hepatic parenchyma to conduct biopsy of a small specimen (about $100 \mathrm{mg}$ ). The animal related procedures used in this experiment were approved by the Animal Care and Use Committee of the National Agricultural Research Center for Hokkaido Region (Sapporo, Japan). The regulations are in conformity with the Guide for the Care and Use of Agricultural Animals in Agricultural Research and Teaching (Federation of Animal Science Societies, 1988).

The thawed liver samples were minced and homogenized with 4 volumes of $0.15 \mathrm{M} \mathrm{KCl}$ solution containing $1 \mathrm{mM}$ EDTA. The homogenate was centrifuged for $15 \mathrm{~min}$ at $9,000 \mathrm{~g}$, and the supernatant fraction was centrifuged at $105,000 \mathrm{~g}$ for $60 \mathrm{~min}$ to obtain the microsomes. The microsomes were then suspended in $0.15 \mathrm{M} \mathrm{KCl}$ solution containing $1 \mathrm{mM}$ EDTA. The protein concentration was determined by the method of Lowry et al. [6] using bovine serum albumin as a standard.

We assayed the UDP-glucuronosyltransferase activity toward estradol- $17 \beta$ in $10 \mu \mathrm{lmicrosomes}(0.025 \mathrm{mg}$ protein/ $\mathrm{m} l$ microsome; activated previously by $0.01 \%$ purified cholate [3]) in $200 \mu \mathrm{L}$ of reaction mixture solution [50 mM Tris-HCl buffer ( $\mathrm{pH}$ 7.4), $3 \mathrm{mM}$ UDP-glucuronic acid (Nakarai Yakuhin Co., Kyoto, Japan), $0.5 \mathrm{mM} \mathrm{MgCl}_{2}$, and $0.25 \mathrm{mM}$ estradiol-17 $\beta$ (Sigma, St. Louis, MO, U.S.A.)] at $37^{\circ} \mathrm{C}$ for $10 \mathrm{~min}$ when the enzyme reactions were within the linear phase $(0.49,0.47,0.47,0.47,0.47,0.41$, and 0.23 $\mathrm{nmol} / \mathrm{min} / \mathrm{mg}$ protein at $2.5,5,10,15,20,30$, and $60 \mathrm{~min}$, respectively). The reaction was stopped by double-boiling at $100^{\circ} \mathrm{C}$. We filtered the resultant enzyme reaction products using a disposable disk filter (HPLC-DISK 3; Kanto
Chemical Co., Tokyo, Japan) and analyzed them using an HPLC system (CR8A-LC10AT-SPD10A-CTO10ASDGU12A, Shimadzu Corporation, Kyoto, Japan) and a Tosoh TSKgel $80 \mathrm{Tm}$ reversedphase column as an analytical column $(7.8 \mathrm{~mm} \times 30 \mathrm{~cm}$; Tosoh Corporation, Tokyo, Japan). The filtrated samples were injected and eluted with an acetonitrile-water-acetic acid (35:65:0.1 v:v:v) solution while monitoring the absorbance at $221 \mathrm{~nm}(1 \mathrm{ml} / \mathrm{min}$ for flow speed at $40^{\circ} \mathrm{C}$ ). These conditions were utilized to analyze the UDP-glucuronosyltransferase activities of microsomes prepared from the rat liver in a previous study [10]. The amounts of two types of glucuronidated estradiol, estradiol-3 $\alpha$-glucuronide and estradiol-17 $\beta$-glucuronide, were determined using the respective authentic standards (E2127 and E1127, Sigma, St. Louis, MO, U.S.A.). Figure 2 a shows the clearly separated peaks of estradiol- $3 \alpha$-glucuronide, estradiol-17 $\beta$-glucuronide and estradiol-17 $\beta$ in a typical HPLC chromatogram of the mix solution.

The parameters of the kinetics of UDP-glucuronosyltransferase for estradol-17 $\beta$, maximum velocity, and Michaelis constant values were calculated from the activity toward a range of estradiol- $17 \beta$ concentrations (from 0.002 to $1 \mathrm{mM}$; Fig. 3) using the Eadie-Hofstee plot and Levenberg-Marquardt nonlinear least-squares algorithm. These were $0.50 \mathrm{nmol} / \mathrm{min} / \mathrm{mg}$ protein and $0.011 \mathrm{mM}$, respectively. The inter- and intra-assay CVs based on 4 replicates of triplicate analysis using the same microsomes were only $2.2 \%$ and $1.8 \%$, respectively. In the euthanized cow, the microsomal UDP-glucuronosyltransferase activity of the biopsy specimen (B; $0.48 \mathrm{nmol} / \mathrm{min} / \mathrm{mg}$ protein) was very similar to that of the sample collected under visual observation (S; $0.47 \mathrm{nmol} / \mathrm{min} / \mathrm{mg}$ protein), and the ratio of $\mathrm{S}$ to $\mathrm{B}$ 


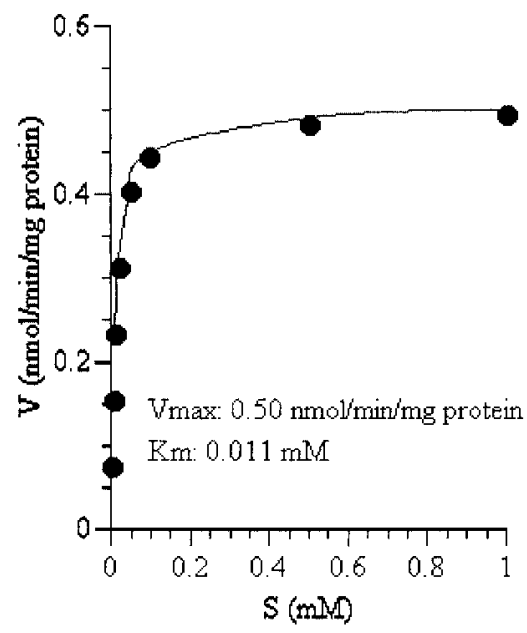

Fig. 3. Relationship between the concentration of estradiol- $17 \beta$ substrate (S) and the reaction speed (v) of estradiol glucuronidation catalized by UDP-glucuronosyltransferases in the microsomes prepared using a liver sample from the euthanized cow.

was $98.1 \%$. The intra-biopsy $\mathrm{CV}$ using the microsome of the second cow was $7.8 \%(n=5)$.

The results of this small-scale study suggest that this method is reliabile. Thus, this method needs to be evaluated further in a larger-scale clinical study. The UDP-glucuronosyltransferase family is a group of complex enzymes that protect organs from various xenobiotics and excess endobiotics, including estradiol-17 $\beta$ [5]. Thus, each enzyme belonging to this family must be respectively controlled according to the changes in the amount of various xenobiotics and endobiotics, which may be affected by changes in the environment, feed management, and physiological conditions.

The importance of the amount of glucuronic acid in the liver of dairy cows may also need to be considered, although no information is currently available from previous studies. Metabolic status and oxidative stress may affect the amount of the glucuronic acid in the liver and the speed of hepatic glucuronidation of estradiol- $17 \beta$ because glucuronic acid is the product of UDP-glucose catalyzed by UDP-glucosedehydrogenase [2] and because glucuronic acid is the precursor for vitamin C [11]. Biopsy specimens may also be used to measure glucuronic acid.

When we compared the HPLC chromatogram of the reac- tion products of estradiol glucuronidation catalyzed by the microsomes of one of the dairy cows (Fig. 2b) with that of a male rat [10], we found an interesting species difference. The hepatic microsomes of the dairy cow produced estradiol-3 $\alpha$-glucuronide as the main product and only subtle amounts of estradiol-17 $\beta$-glucuronide, whereas the hepatic microsomes of the rat produce both and estradiol- $17 \beta$-glucuronide is the main product. We were only able to find a small peak of estradiol-17 $\beta$-glucuronides in an HPLC chromatogram when the linear phase enzyme reactions were complete and the reaction was allowed to continue for more than $60 \mathrm{~min}$ (data not shown). Thus, the liver of dairy cows also produced this conjugate, but at a very slow speed. Therefore, dairy cows may have some unique enzymes among the UDP-glucuronosyltransferase family.

In conclusion, the results of this study suggest that this method of using a combination of hepatic biopsy and in vitro glucuronidation followed by the HPLC analysis is reliable. Moreover, this study suggests that this method may become a new tool for use in clinical studies.

ACKNOWLEDGEMENTS. We are grateful to Prof. Hiroshi Satoh and Dr. Tharwat Mohamed of Rakuno Gakuen University for their important advice concerning hepatic biopsy.

\section{REFERENCES}

1. Agriculture, Forestry and Fisheries Research Council Secretariat. 1999. Japanese Feeding Standard for Dairy Cattle. Central Association of Livestock Industry, Tokyo, Japan.

2. Beylot, M., Peroni, O., Diraison, F. and Large, V. 1997. Diabetes Metab. 23: 251-257.

3. Imai, Y. 1979. J. Biochem. 86: 1697-1707.

4. Kadokawa, H. and Martin, G. B. 2006. J. Reprod. Dev. 52: 161-168.

5. King, C. D., Rios, G. R., Green, M. D. and Tephly, T. R. 2000. Curr. Drug. Metab. 1: 143-161.

6. Lowry, O. H., Rosebrough, N.J., Farr, A. L. and Randall, R. J. 1951. J. Biol. Chem. 193: 265-275.

7. Martin, G. B. and Kadokawa, H. 2006. J. Reprod. Dev. 52: 145-152.

8. Matsumoto, J., Yokota, H. and Yuasa, A. 2002. Environ. Health Perspect. 110: 193-196.

9. Mohamed, T., Oikawa, S., Nakada, K., Kurosawa, T., Sawamukai, Y. and Sato, H. 2003. J. Vet. Med. Sci. 65: 821-824.

10. Shibata, N., Matsumoto, J., Nakada, K., Yuasa, A. and Yokota, H. 2002. Biochem. J. 368: 783-788.

11. Smirnoff, N. 2001. Vitam. Horm. 61: 241-266.

12. Wiltbank, M., Lopez, H., Sartori, R., Sangsritavong, S. and Gumen, A. 2006. Theriogenology 65: 17-29. 